\title{
The Nursing Home Pneumonia Risk Index: A Simple, Valid MDS-Based Method of Identifying 6-Month Risk for Pneumonia and Mortality
}

\author{
Philip D. Sloane MD, MPH ${ }^{\mathrm{a}, \mathrm{b}, *}$, Sheryl Zimmerman $\mathrm{PhD}^{\mathrm{a}, \mathrm{c}}$, Kimberly Ward BA ${ }^{\mathrm{a}}$, \\ David Reed $\mathrm{PhD}^{\mathrm{a}}$, John S. Preisser PhD ${ }^{\mathrm{d}}$, David J. Weber MD, MPH ${ }^{\mathrm{e}, \mathrm{f}}$ \\ ${ }^{a}$ Cecil G. Sheps Center for Health Services Research, University of North Carolina at Chapel Hill, Chapel Hill, NC \\ ${ }^{\mathrm{b}}$ Department of Family Medicine, University of North Carolina at Chapel Hill, Chapel Hill, NC \\ ' School of Social Work, University of North Carolina at Chapel Hill, Chapel Hill, NC \\ ${ }^{\mathrm{d}}$ Gillings School of Global Public Health, University of North Carolina at Chapel Hill, Chapel Hill, NC \\ e Division of Infectious Diseases, Department of Medicine, School of Medicine, University of North Carolina at Chapel Hill, Chapel Hill, NC \\ ${ }^{\mathrm{f}}$ Department of Epidemiology, School of Public Health, University of North Carolina at Chapel Hill, Chapel Hill, NC
}

\section{A B S T R A C T}

Keywords:

Long-term care

nursing homes

pneumonia risk

infections

mortality
Background: Pneumonia is the leading infectious cause of hospitalization and death for nursing home $(\mathrm{NH})$ residents; however, diagnosis is often delayed because classic signs of infection are not present. We sought to identify $\mathrm{NH}$ residents at high risk for pneumonia, to identify persons to target for more intensive surveillance and preventive measures.

Methods: Based on a literature review, we identified key risk factors for pneumonia and compiled them for use as prediction tool, limiting risk factors to those available on the Minimum Data Set (MDS). Next, we tested the tool's ability to predict 6-month pneumonia incidence and mortality rates in a sample of 674 residents from $7 \mathrm{NHs}$, evaluating it both as a continuous and a dichotomous variable, and applying both logistic regression and survival analysis to calculate estimates.

Results: NH Pneumonia Risk Index scores ranged from -1 to 6 , with a mean of 2.1, a median of 2, and a mode of 2 . For the outcome of pneumonia, a 1-point increase in the index was associated with a risk odds ratio of $1.26(P=.038)$ or a hazard ratio of $1.24(P=.037)$; using it as a dichotomous variable $(\leq 2 \mathrm{vs} \geq 3)$, the corresponding figures were a risk odds ratio of $1.78(P=.045)$ and a hazard ratio of $1.82(P=.025)$. For the outcome of mortality, a 1-point increase in the NH Pneumonia Risk Index was associated with a risk odds ratio of $1.58(P=.002)$ and a hazard ratio of $1.45(P=.013)$; using the index as a dichotomous variable, the corresponding figures were a risk odds ratio of $3.71(P<.001)$ and a hazard ratio of 3.29 $(P=.001)$.

Conclusions: The NH Pneumonia Risk Index can be used by NH staff to identify residents for whom to apply especially intensive preventive measures and surveillance. Because of its strong association with mortality, the index may also be valuable in care planning and discussion of advance directives.
Pneumonia is the leading infectious cause of hospitalization and death for nursing home $(\mathrm{NH})$ residents. ${ }^{1,2}$ Estimates of $\mathrm{NH}$ pneumonia incidence range from 0.3 to 2.5 cases per 1000 resident-days, ${ }^{3-5}$ figures that are nearly 10 times the incidence rate for older persons living

This research was supported by grant R01HS022298 from the Agency for Healthcare Research and Quality.

The authors declare no conflicts of interest.

* Address correspondence to Philip D. Sloane, MD, MPH, Cecil G. Sheps Center for Health Services Research University of North Carolina at Chapel Hill, 725 Martin Luther King Jr. Boulevard, Chapel Hill, NC 27599-7590.

E-mail address: Philip_Sloane@med.unc.edu (P.D. Sloane). in the community. ${ }^{4}$ Related hospitalization rates are also much higher than for older adults living in the community; in fact, $\mathrm{NH}$ residents account for $10 \%$ to $18 \%$ of all people hospitalized for pneumonia. ${ }^{6}$

$\mathrm{NH}$-acquired pneumonia has the highest mortality among $\mathrm{NH}$-acquired infections. The case fatality rate is around $25 \%$, in part because pneumonia differentially affects $\mathrm{NH}$ residents who are frail and have multiple comorbidities. ${ }^{2}$ Furthermore, $\mathrm{NH}$ residents diagnosed with pneumonia have a 1-year all-cause mortality rate that is approximately twice that of a matched comparison group without pneumonia. ${ }^{7}$ Unfortunately, in the high-risk, chronically ill NH population, a pneumonia diagnosis is often delayed because classic signs 
of infection, such as fever, cough, and mental status change, are frequently absent. 8,9

A variety of risk factors for pneumonia have been identified. Prominent among these include advanced age, male gender, chronic pulmonary or cardiac disease, antipsychotic or other sedative medication use, advanced dementia, swallowing difficulties, impaired functional status, and poor oral health., ${ }^{1,10-15}$ According to one review, the main risk factors for $\mathrm{NH}$ pneumonia tend to be fairly stable and uniform across multiple studies and to differ markedly from the risk factors for community-acquired pneumonia. ${ }^{4}$

As part of an intervention trial of the impact of enhanced oral hygiene on pneumonia incidence in $\mathrm{NHs}$, we wanted to identify residents at high risk for pneumonia, thereby helping nursing staff prioritize the deployment of a dedicated oral care aide. A review of the literature found numerous studies identifying specific risk factors but no validated instrument to quantify pneumonia risk. Therefore, we sought to develop and validate a pneumonia risk scorecard using commonly available demographic and health status variables.

\section{Methods}

Using the risk score development methods of Charlson et al, ${ }^{16}$ Sloane et $\mathrm{al}^{17}{ }^{17}$ and Berlowitz et $\mathrm{al}^{18}{ }^{18}$ our study team, consisting of a geriatrician (P.D.S.), epidemiologist and $\mathrm{NH}$ health services researcher (S.Z.), infectious disease epidemiologist (D.W.), analyst (D.R.), and biostatistician (J.P.) reviewed the literature on pneumonia, including aspiration pneumonia, with the goal of developing a simple prediction tool to quantify each resident's pneumonia risk. To make the risk assessment tool feasible to calculate on all $\mathrm{NH}$ residents, we limited risk factors to those readily available on the Minimum Data Set (MDS), a standardized evaluation that is completed for all $\mathrm{NH}$ residents on an annual basis and updated at least quarterly. ${ }^{19-21}$

Significant risk factors for $\mathrm{NH}$-acquired pneumonia identified in prior reports included aspiration (odds risk [OR] 13.9), inability to take oral medication (OR 8.3), sedative medication use (OR 2.6), bedbound (OR 2.5), swallowing difficulty (OR 2.0), male gender (OR 1.9), older age (OR 1.7 per 10 years), and increased morbidity (OR 1.2). Independent predictors of aspiration pneumonia in $\mathrm{NH}$ residents include suctioning (OR 2.6), chronic obstructive pulmonary disease (OR 2.5), congestive heart failure (OR 1.8), feeding tube (OR 1.7), bedfast (OR 1.7), delirium/less alertness (OR 1.6), weight loss (OR 1.6), swallowing problem/dysphagia (OR 1.5), urinary tract infection (OR 1.4), mechanically altered diet (OR 1.2), dependence in eating (OR 1.2), and the following factors that were identified as risk factors for infection without an identified odds or risk ratio: poor oral hygiene, tracheostomy care, number of comorbid conditions, Alzheimer disease, behavioral symptoms of dementia, and a history of gastroesophageal reflux. . $7,10-15,22,23^{-1}$

Risk factors that are readily available in the MDS 3.0 were compiled into the NH Pneumonia Risk Index (see Table 1). Because of varying reports regarding relative strength of many items, each was assigned a score of +1 or -1 . Characteristics included as increasing risk are male gender; age > 85 years; a diagnosis of dementia, chronic lung disease, and chronic renal disease; requiring extensive or total assistance in eating; coughing or choking during meals (a marker for swallowing problems); behavior interfering with care; dental problems (a marker of poor oral hygiene); and current use of a sedative or antipsychotic medication. The single factor decreasing risk was edentulous status (a protective factor for oral hygiene). These items generate an overall risk score ranging from -1 to +10 , with higher scores indicating greater risk.

The NH Pneumonia Risk Index was field tested in 7 NHs in North Carolina, each of which was participating as a control site in an
Table 1

The Nursing Home Pneumonia Risk Index

\begin{tabular}{lll}
\hline Resident Characteristic & $\begin{array}{l}\text { Minimum Data Set } \\
\text { (MDS) 3.0 Item }\end{array}$ & Points \\
\hline Male gender & A0800 & +1 \\
Age $\geq 85$ y & A0900 & +1 \\
Diagnosis: Alzheimer disease/dementia & I14200 or I14800 & +1 \\
$\quad$ Chronic lung disease & I6200 & +1 \\
$\quad$ Chronic renal disease & K0100C & +1 \\
Requires extensive or total assistance & G0110H & +1 \\
$\quad$ in eating & K0100C & +1 \\
Noted to have coughing or choking & & \\
$\quad$ during meals & E0500B or E0800 & +1 \\
Behavior interferes with care & L0200 (not B or Z) & +1 \\
Dental problems & L0200B & -1 \\
No teeth & N0400 A, B, or D & +1 \\
On a sedative or antipsychotic medication & &
\end{tabular}

intervention study. Among the sample NHs, 5 (71\%) were for-profit, the mean bed size was 106 (range 62-134), and the mean score on the overall $\mathrm{NH}$ quality score in Nursing Home Compare (which ranges from 0 to 5) was 3.1. ${ }^{24}$ Medical records were audited on all 720 residents during site visits conducted between October 2014 and April 2016, and each resident was followed for 6 months or until death or discharge from the $\mathrm{NH}$; incident pneumonia or mortality were recorded for all residents for up to 6 months. Of the cases audited, 46 were excluded from analysis because MDS data used to calculate the NH Pneumonia Risk Index were unavailable or more than 4 months out of date, yielding a final sample size of 674 . All study procedures were approved by the Institutional Review Board of the University of North Carolina at Chapel Hill.

A positive case of pneumonia was identified based on written documentation of the diagnosis by a physician, nurse practitioner, physician assistant, or licensed nurse in the $\mathrm{NH}$ record or a hospital discharge summary, a method found to be highly sensitive when compared to detecting pneumonia based on recorded signs and symptoms or prescription of an antibiotic. ${ }^{9}$ Mortality was confirmed based on a written note in the medical record with the date of death.

Data were double-entered using Microsoft Access and analyses conducted in SPSS, version 24. Descriptive statistics were used to calculate measures of central tendency, Cronbach alpha, and distributions of the variables of interest. To explore the relationship between NH Pneumonia Risk Index scores and 6-month pneumonia incidence and mortality, risk scores were examined as both a continuous variable and as a dichotomous variable $(\leq 2$ and $\geq 3$ ), applying both logistic regression (GENLIN command) and survival analysis (COXREG command) to estimate $P$ values. $\mathrm{NH}$ site was added as a covariate to adjust for variation among sites.

\section{Results}

The NH Pneumonia Risk Index scores among the 674 NH residents in the study sample ranged from -1 to 6 , with a mean of 2.1 (standard deviation 1.3), a median of 2, and a mode of 2. As shown in Table 2, scores of 1,2 , and 3 were documented for $25 \%, 30 \%$, and $21 \%$ of the sample, respectively; $65 \%$ of the sample scored $\leq 2$, and $35 \%$ scored $\geq 3$. Cronbach alpha for the measure was 0.1, confirming that it functions more as an index than as a scale.

Table 2 displays the relationship between baseline NH Pneumonia Risk Index scores and the 6-month incidence of pneumonia and mortality. The 6-month pneumonia incidence rate ranged from $3 \%$ for scores of -1 or 0 , to $13 \%$ for scores of 4 or 5 . Mortality rate ranged from $1 \%$ for scores of -1 or 0 to $19 \%$ for a score of 5 . When index scores were dichotomized, a score of $\leq 2$ was associated with a pneumonia 
Table 2

Rates of Pneumonia and Death Within 6 Months After Pneumonia Risk Assessment

\begin{tabular}{lccccc}
\hline $\begin{array}{l}\text { Pneumonia } \\
\text { Risk Score }\end{array}$ & $\begin{array}{l}\text { Number (\%) } \\
\text { of Residents }\end{array}$ & $\begin{array}{l}\text { Number of } \\
\text { Cases of } \\
\text { Pneumonia }\end{array}$ & $\begin{array}{l}\text { Pneumonia } \\
\text { Incidence } \\
\text { Rate, \% }\end{array}$ & $\begin{array}{l}\text { Number of } \\
\text { Deaths }\end{array}$ & $\begin{array}{l}\text { Mortality } \\
\text { Rate, \% }\end{array}$ \\
\hline-1 or 0 & $69^{*}(10)$ & 2 & 3 & 1 & 1 \\
1 & $166(25)$ & 14 & 8 & 4 & 2 \\
2 & $204(30)$ & 13 & 6 & 8 & 4 \\
3 & $144(21)$ & 16 & 11 & 18 & 13 \\
4 & $72(11)$ & 9 & 13 & 1 & 1 \\
5 & $16(2)$ & 2 & 13 & 3 & 19 \\
$\geq 6$ & $3(<1)$ & 0 & 0 & 0 & 0 \\
$\leq 2$ & $439(65)$ & 29 & 6.6 & 13 & 3.0 \\
$\geq 3$ & $235(35)$ & 27 & 11.5 & 22 & 9.4 \\
Total & 674 & 56 & 8 & 35 & 5 \\
\hline
\end{tabular}

*Three had a score of $-1 ; 66$ had a score of 0 .

incidence rate of $6.6 \%$ and a mortality rate of $3.0 \%$, whereas scores $\geq 3$ were associated with a pneumonia incidence rate of $11.5 \%$ and a mortality rate of $9.4 \%$.

For the outcome of pneumonia, using the NH Pneumonia Risk Index as a continuous variable, a 1-point increase in the index was associated with a risk odds ratio of $1.26(P=.038)$ or a hazard ratio of $1.24(P=.037)$ for pneumonia within 6 months; using it as a dichotomous variable ( $\leq 2 \mathrm{vs} \geq 3$ ), the corresponding figures were a risk odds ratio of $1.78(P=.045)$ and a hazard ratio of $1.82(P=.025)$. For the outcome of mortality, a 1-point increase in the NH Pneumonia Risk Index was associated with a risk odds ratio of $1.58(P=.002)$ and a hazard ratio of $1.45(P=.013)$; using the index as a dichotomous variable, the corresponding figures were a risk odds ratio of 3.71 $(P<.001)$ and a hazard ratio of $3.29(P=.001)$.

\section{Discussion}

Nursing homes are under increasing pressure to prevent hospitalization due to pneumonia and other acute, potentially preventable health events. ${ }^{25}$ However, because resources are limited, it is important to identify persons who are at particularly high risk, so that preventive interventions can be targeted toward those most likely to benefit. This research sought to develop and validate a simple method of identifying persons at increased risk for pneumonia who could benefit from such preventive efforts as mobilization, enhanced oral hygiene care, and additional medical scrutiny. ${ }^{26}$

Using variables obtained from the MDS-a routine nursing screening tool mandated by law for all federally certified $\mathrm{NHs}$, the $\mathrm{NH}$ Pneumonia Risk Index identifies a subgroup of one-third of NH residents whose 6-month risk of pneumonia is nearly twice that of the others (Table 2). Thus, NH staff may wish to compute the index to identify residents who need especially aggressive management to prevent pneumonia and identify respiratory symptoms consistent with pneumonia early, so that incipient pneumonia can potentially be treated without requiring hospitalization.

Although the index was not developed to assess mortality, a serendipitous finding was that the $\mathrm{NH}$ Pneumonia Risk Index also estimates 6-month mortality, and that indeed its association with mortality is even greater than its association with pneumonia (Table 2). Therefore, the NH Pneumonia Risk Index may also be useful when applied to estimate mortality risk, a factor that can be valuable in care planning and discussion of advance directives.

A potential limitation of these results is the possibility of mislabeling. Despite the fact that the MDS has been extensively validated, errors or omissions are inevitable when such an extensive, standardized assessment tool is routinely collected on persons whose verbal and cognitive abilities are often limited. ${ }^{19,27}$ Furthermore, pneumonia is difficult to diagnose and is likely misdiagnosed frequently, even in hospital settings, as cases with the diagnosis may have negative chest radiographs, negative examinations, lack of cough or fever, and negative blood cultures. ${ }^{28}$ Consequently, some diagnostic mislabeling may have been present in these data. That said, the data represent what is standard in $\mathrm{NH}$ practice, and what is available for regulatory, quality assurance, and quality improvement activities in community NH settings. Moreover, the score is easy to derive, and potential mislabeling notwithstanding, identifies residents at high risk of pneumonia and mortality.

Finally, additional validation of the NH Pneumonia Risk Index is indicated. Although the study NHs were typical of US NHs, more widespread field testing would help identify whether and to what extent there is variation in the index's predictive power in other $\mathrm{NH}$ settings. As part of these efforts, it may be useful to compare the index with the measure recently developed by Jackson and colleagues for pneumonia risk among community elderly. ${ }^{29}$

\section{References}

1. Beck-Sague C, Banerjee S, Jarvis WR. Infectious diseases and mortality among nursing home residents. Am J Public Health 1993;83:1739-1742.

2. Dhawan N, Pandya N, Khalili M, et al. Predictors of mortality for nursing homeacquired pneumonia: A systematic review. Biomed Res Int 2015;2015:285983.

3. Mehr DR, Binder EF, Kruse RL, et al. Predicting mortality in nursing home residents with lower respiratory tract infection: The Missouri LRI Study. JAMA $2001 ; 286: 2427-2436$

4. Loeb MB. Pneumonia in nursing homes and long-term care facilities. Semin Respir Crit Care Med 2005;6:650-655.

5. Jones AL, Dwyer LL, Bercovitz AR, et al. The National Nursing Home Survey: 2004 overview. Vital Health Stat 2009;167:1-155.

6. Montoya A, Mody L. Common infections in nursing homes: A review of current issues and challenges. Aging Health 2011;7:889-899.

7. Vergis EN, Brenned C, Ageener M, et al. Pneumonia in long-term care: A prospective case-control study of risk factors and impact on survival. Arch Intern Med 2001;161:2378-2381.

8. Janssens JP, Krause KH. Pneumonia in the very old. Lancet Infect Dis 2004;4: $112-124$.

9. Zimmerman S, Cohen LW, Scales K, et al. Pneumonia identification using nursing home records. Res Gerontol Nurs 2015;28:1-6.

10. El-Solh AE, Niederman MS, Drinka P. Nursing home-acquired pneumonia: A review of risk factors and therapeutic approaches. Curr Med Res Opin 2010;26: 2707-2714.

11. Loeb M, McGeer A, McArthur M, et al. Risk factors for pneumonia and other lower respiratory tract infections in elderly residents of long-term care facilities. Arch Intern Med 1999;159:2058-2064.

12. Magaziner J, Tenney JH, DeForge B, et al. Prevalence and characteristics of nursing-home acquired infections in the aged. J Am Geriatr Soc 1991;39: 1070-1078.

13. Jacobson C, Strausbaugh LJ. Incidence and impact of infection in the nursing home care unit. Am J Infect Control 1990;18:151-159.

14. Setia U, Serventi I, Lorenz P. Nosocomial infections among patients in the longterm care facility: Spectrum, prevalence, and risk factors. Am J Infect Control $1998 ; 13: 57-62$.

15. Rantanen TK, Salo JA. Gastroesophageal reflex disease as a cause of death Analysis of fatal cases under conservative treatment. Scand J Gastroenterol 1999;34:229-233.

16. Charlson ME, Pompei P, Ales KL, et al. A new method of classifying prognostic comorbidity in longitudinal studies: Development and validation. J Chronic Dis 1987; 40:373-383.

17. Sloane P, Ivey J, Roth M, et al. Accounting for the sedative and analgesic effects of medication changes during patient participation in clinical research studies: Measurement development and application to a sample of institutionalized geriatric patients. Contemp Clin Trials 2008;29:140-148.

18. Berlowitz DR, Brandeis GH, Morris JN, et al. Deriving a risk-adjustment model for pressure ulcer development using the Minimum Data Set. J Am Geriatr Soc 2001;49:866-871.

19. Hawes C, Morris JN, Phillips CD, et al. Development of the nursing home Resident Assessment Instrument in the USA. Age Ageing 1997:26:S19-S25.

20. Morris JN, Hawes C, Fries BE, et al. Designing the National Resident Assessment Instrument for nursing homes. Gerontologist 1990;30:293-307.

21. Morley JE. Minimum Data Set 3.0: A giant step forward. J Am Med Dir Assoc $2013 ; 14: 1-3$

22. Langmore SE, Skarupski KA, Park PS, et al. Predictors of aspiration pneumonia in nursing home residents. Dysphagia 2002;17:298-307.

23. van der Steen JT, Ooms ME, Mehr DR, et al. Severe dementia and adverse outcomes of nursing home-acquired pneumonia: Evidence for mediation by functional and pathophysiological decline. J Am Geriatr Soc 2002;50:439-448. 
24. Center for Medicare and Medicaid Services. Nursing Home Compare. Available at: https://www.medicare.gov/nursinghomecompare. Accessed February 9, 2017.

25. Ouslander JG, Maslow K. Geriatrics and the triple aim: Defining preventable hospitalizations in the long-term care population. J Am Geriatr Soc 2012;60: 2313-2318.

26. Loeb $\mathrm{MB}$, Becker $\mathrm{M}$, Eady $\mathrm{A}$, et al. Interventions to prevent aspiration pneumonia in older adults: A systematic review. J Am Geriatr Soc 2003;51: 1018-1022.
27. Cai S, Mukamel DB, Veazie P, et al. Validation of the Minimum Data Set in identifying hospitalization events and payment source. J Am Med Dir Assoc 2011;12:38-43.

28. Ayaz SI, Haque N, Pearson C, et al. Nursing home-acquired pneumonia: Course and management in the emergency department. Int J Emerg Med 2014;12: 7-19.

29. Jackson ML, Walker $\mathrm{R}$, Lee $\mathrm{S}$, et al. Predicting 2-year risk of developing pneumonia in older adults without dementia. J Am Geriatr Soc 2016;64: 1439-1447. 